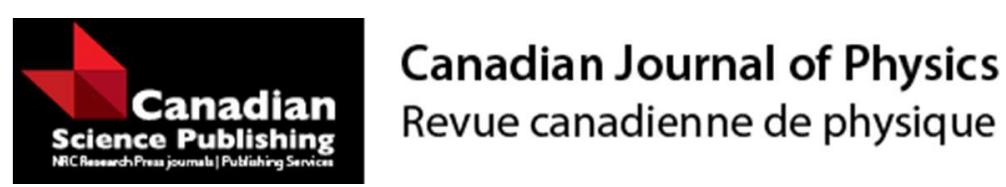

\title{
Kantowski-Sachs scalar field cosmological models in a modified theory of gravity
}

\begin{tabular}{|r|l|}
\hline Journal: & Canadian Journal of Physics \\
\hline Manuscript ID & cjp-2016-0718 \\
\hline Manuscript Type: & Article \\
\hline Date Submitted by the Author: & 03-Oct-2016 \\
\hline Complete List of Authors: & $\begin{array}{l}\text { M., Vijaya Santhi; Andhra University, Applied Mathematics } \\
\text { RAO, V.U.M. ; Andhra University, Applied Mathematics } \\
\text { Aditya, Y.; Andhra University, Applied Mathematics }\end{array}$ \\
\hline Keyword: & $\begin{array}{l}\text { Kantowski-Sachs model, f(R, T) gravity, Scalar field cosmology,, Modified } \\
\text { gravity, Cosmology }\end{array}$ \\
\hline
\end{tabular}




\title{
Kantowski-Sachs scalar field cosmological models in a modif ied theory of gravity
}

\author{
M. Vijaya Santhi, V.U.M. Rao, Y. Aditya \\ Department of Applied Mathematics, Andhra University, Visakhapatnam-530003, India \\ gv.santhi@live.com
}

\begin{abstract}
In this paper, we investigate anisotropic Kantowski-Sachs model in $f(R, T)$ theory of gravity proposed by Harko et al. (Physics review D 84, 024020, 2011) with scalar field (quintessence or phantom). Here $R$ is Ricci scalar and $T$ is the trace of energy momentum tensor. The field equations have been solved using the fact that scalar expansion is proportional to the shear scalar of the space-time. We explore the behavior of deceleration parameter, which represents a transition of the universe from early decelerating phase to present accelerated phase. Some physical properties and various cosmological distance measures are also obtained and discussed.
\end{abstract}

Key words: Kantowski-Sachs model, $f(R, T)$ gravity, Scalar field cosmology, , Modified gravity.

\section{Introduction:}

Modern observational data on the cosmic expansion history (Perlmutter et al. [1]; Spergel et al. [2]; Tegmark et al. [3]; Riess et al. [4]; Fedeli et al. [5]) has lead to the discovery of accelerated expansion of the universe. It is believed that the reason for this is an exotic type of unknown force with huge negative pressure dubbed as dark energy (DE). However, the nature and behavior of DE is still a mystery. Currently, there are two main approaches for the explanation of this accelerated expansion. One way is to introduce scalar field models like phantom (Caldwell [6]; Nojiri and Odintsov [7]), quintessence (Sahni and Starobinsky [8]; Sahni [9]; Padmanabhan [10]), anisotropic fluids (Akarsu and Kilinc [11]; Sharif and Zubair [12]) and etc. An alternative approach is to modify Einstein-Hilbert action to obtain alternate theories of gravity like $f(R)$ theory of gravity which provides a natural unification of early-time inflation and late-time acceleration (Capozziello and Francaviglia [13]; Nojiri and Odintsov [14]). Among the other modified theories, theory of scale-Gauss-Bonnet gravity, so called $f(G)$ gravity (Nojiri and Odintsov [15]) and $f(T)$ gravity (Linder [16]), where $T$ is the torsion have been proposed to explain the accelerated expansion of universe.

Recently, Harko et al. [17] proposed another modified theory known as $f(R, T)$ gravity, wherein the gravitational Lagrangian contains the Ricci scalar $R$ and trace of the energy-momentum tensor $T$. It is well known that in the study of early stages of evolution of the universe anisotropic models 
play a vital role. Adhav [18] has obtained LRS Bianchi type- $I$ cosmological model in $f(R, T)$ gravity. Reddy and Santhi Kumar [19] have discussed some anisotropic cosmological models in $f(R, T)$ theory of gravity. Mishra and Sahoo [20] have studied Bianchi type- $V I_{h}$ perfect fluid cosmological model in $f(R, T)$ gravity. Rao and Neelima [21] have obtained perfect fluid Bianchi type- $V I_{0}$ universes in $f(R, T)$ gravity. Rao et al. [22] have investigated Bianchi type- $I I, V I I I$ and $I X$ cosmological models in $f(R, T)$ theory of gravity. Houndjo [23] has developed the cosmological reconstruction of $f(R, T)$ gravity and discussed the transition of matter dominated phase to an accelerated phase. Shri Ram and Chandel [24] have discussed dynamics of magnetized string cosmological model in $f(R, T)$ theory of gravity. Rao et al. [25] have obtained anisotropic Bianchi type- $V I_{h}$ perfect fluid cosmological models in $f(R, T)$ theory of gravity. Aditya et al. [26] have studied Bianchi type- $I I, V I I I$ and $I X$ cosmological models in $f(R, T)$ theory of gravity with variable $\Lambda$.

Scalar fields play a crucial role in particle physics and cosmology. Olive [27] has shown that, during inflation, the potential of a scalar field acts as a dynamical vacuum energy. This prominent role of scalar fields is also evident in models proposed to explain the late time accelerated expansion of the universe in vacuum energy and in evolving quintessence models ([28]-[30]). Further, it was recently proposed that a scalar field can also be the source of the anomalous acceleration [31]. Sharif and Zubair [32] have investigated the anisotropic universe models in $f(R, T)$ gravity in the presence of perfect fluid and scalar field. Singh and Singh [33] have obtained the FriedmannRobertson-Walker (FRW) models with perfect fluid and scalar field in higher derivative theory. Sharif and Jawad [34] have studied reconstruction of scalar field dark energy models in KaluzaKlein universe. Singh and Singh [35] have discussed the behavior of scalar field in modified $f(R, T)$ gravity within the framework of a flat FRW cosmological model. Later, Singh et al. [36] have investigated Bianchi type-I universe with scalar field and time varying cosmological constant in $f(R, T)$ gravity. Recently, Samanta [38] and Reddy et al. [39] have investigated Kantowski-Sachs cosmological models in $f(R, T)$ theory of gravity.

Motivated by the above investigations, we study in this paper Kantowski-Sachs space time in presence of scalar field within the framework of $f(R, T)$ theory of gravitation proposed by Harko et al. [17]. Very recently, Singh et al. [36] have obtained Bianchi type- $I$ scalar field cosmological models in $f(R, T)$ theory, using the power law and exponential law for scalar potentials, we discuss Kantowski-Sachs scalar field cosmological model in this theory without using power law or exponential law for scalar potentials. The plan of the work as follows: Sect. 2 describes $f(R, T)$ gravity formalism in the presence of scaler field. Sect. 3 is devoted to the derivation of field equations and solutions of field equations leading to scalar field model. Sect. 4 contains a detailed physical discussion of the model. Summery and conclusions are presented in the last section. 


\section{$2 f(R, T)$ gravity formalism with scalar field:}

The field equations of $f(R, T)$ gravity are derived from the Hilbert-Einstein type variation principle. The action for the $f(R, T)$ gravity with scalar field is [35]

$$
S=\frac{1}{16 \pi} \int f(R, T) \sqrt{-g} d^{4} x+\int L_{\phi} \sqrt{-g} d^{4} x,
$$

where $f(R, T)$ is an arbitrary function of Ricci scalar $R, T$ is the trace of stress-energy tensor $\left(T_{i j}\right)$ of the matter and $L_{\phi}$ is the matter Lagrangian of scalr field.

The energy momentum tensor $T_{i j}$ is defined as

$$
T_{i j}=-\left(\frac{2}{\sqrt{-g}}\right) \frac{\delta(\sqrt{-g}) L_{\phi}}{\delta g^{i j}} .
$$

Here we consider that the dependence of matter Lagrangian is merely on the metric tensor $g_{i j}$ rather than on its derivatives and we obtain

$$
T_{i j}=g_{i j} L_{\phi}-\frac{\partial L_{\phi}}{\partial g^{i j}} .
$$

Now varying the action $S$ with respect to metric tensor $g_{i j}, f(R, T)$ gravity field equations are obtained as

$$
f_{R}(R, T) R_{i j}-\frac{1}{2} f(R, T) g_{i j}+\left(g_{i j} \square-\nabla_{i} \nabla_{j}\right) f_{R}(R, T)=8 \pi T_{i j}-f_{T}(R, T) T_{i j}-f_{T}(R, T) \Theta_{i j}
$$

where

$$
\Theta_{i j}=-2 T_{i j}+g_{i j} L_{\phi}-2 g^{\alpha \beta} \frac{\partial^{2} L_{\phi}}{\partial g^{i j} \partial g^{\alpha \beta}} .
$$

Here $f_{R}(R, T)=\frac{\partial f(R, T)}{\partial R}, f_{T}(R, T)=\frac{\partial f(R, T)}{\partial T}$ and $\square=\nabla^{\mu} \nabla_{\mu}$, where $\nabla_{\mu}$ denotes the covariant derivative.

Here we assume that the universe is filled with scalar field minimally coupled to gravity. Therefore, the energy-momentum tensor of a scalar field $\phi$ with self-interacting scalar field potential $\mathcal{V}(\phi)$ has the form

$$
T_{i j}=\epsilon \phi_{, i} \phi_{, j}-g_{i j}\left(\frac{\epsilon}{2} g^{\mu \nu} \phi_{, \mu} \phi_{, \nu}-\mathcal{V}(\phi)\right)
$$

where $\epsilon= \pm 1$ correspond to quintessence and phantom scalar fields respectively. The trace of the energy-momentum tensor $T=g^{i j} T_{i j}$ is given by

$$
T=-\epsilon \dot{\phi}^{2}+4 \mathcal{V}(\phi)
$$

hereafter dot denotes differentiation with respect to time $t$. The matter Lagrangian of the scalar field is given by

$$
L_{\phi}=-\frac{1}{2} \epsilon \dot{\phi}^{2}+\mathcal{V}(\phi)
$$


Now from equations (5) and (8), we have

$$
\Theta_{i j}=-2 T_{i j}-g_{i j}\left(\frac{1}{2} \epsilon \dot{\phi}^{2}-\mathcal{V}(\phi)\right) .
$$

Generally, the field equations also depend, through the tensor $\Theta_{i j}$, on the physical nature of the matter field. Hence in the case of $f(R, T)$ gravity depending on the nature of the matter source, we obtain several theoretical models corresponding to different matter contributions for $f(R, T)$ gravity. However, Harko et al. [17] gave three classes of these models:

$$
f(R, T)=\left\{\begin{array}{l}
R+2 f(T) \\
f_{1}(R)+f_{2}(T) \\
f_{1}(R)+f_{2}(R) f_{3}(T) .
\end{array}\right.
$$

Here we consider the first case, i.e $f(R, T)=R+f(T)$, where $f(T)$ is an arbitrary function of the trace of stress-energy tensor $T_{i j}$. Using this relation $f(R, T)$ gravity field equations (4) reduced to

$$
R_{i j}-\frac{1}{2} R g_{i j}=T_{i j}-2\left(T_{i j}+\Theta_{i j}\right) f^{\prime}(T)+f(T) g_{i j}
$$

where a prime denotes differentiation with respect to the argument.

\section{Field equations and the scalar field models:}

Here we consider the Kantowski-Sachs space-time in the form

$$
d s^{2}=d t^{2}-A^{2} d r^{2}-B^{2}\left(d \psi^{2}+\sin ^{2} \psi d \varphi^{2}\right)
$$

where $A$ and $B$ are functions of cosmic time $t$ only. Kantowski-Sachs class of metrics represent anisotropic and homogeneous but expanding (or contracting) cosmologies. They also provide models where the effects of anisotropies can be estimated and compared with the FRW class of cosmologies (Thorne, [37]).

For the particular choice of the function $f(T)=\lambda T$ (Harko et al. [17]), where $\lambda$ is a constant, the field equations (10) for the metric (11) using (6) and (9) can be written as

$$
\begin{aligned}
2 \frac{\ddot{B}}{B}+\frac{\dot{B}^{2}}{B^{2}}+\frac{1}{B^{2}} & =\left(\frac{1+2 \lambda}{2}\right) \epsilon \dot{\phi}^{2}-(4 \lambda+1) \mathcal{V}(\phi) \\
\frac{\ddot{A}}{A}+\frac{\ddot{B}}{B}+\frac{\dot{A} \dot{B}}{A B} & =\left(\frac{1+2 \lambda}{2}\right) \epsilon \dot{\phi}^{2}-(4 \lambda+1) \mathcal{V}(\phi) \\
\frac{\dot{B}^{2}}{B^{2}}+2 \frac{\dot{A} \dot{B}}{A B}+\frac{1}{B^{2}} & =-\left(\frac{1+2 \lambda}{2}\right) \epsilon \dot{\phi}^{2}-(4 \lambda+1) \mathcal{V}(\phi)
\end{aligned}
$$

Equations (12)-(14) are a set of three independent equations with four unknowns $(A, B, \phi$ and $\mathcal{V}(\phi))$. Therefore, we need an additional constraint to solve the above system. Here we use the 
physical condition that the expansion scalar $\theta$ is proportional to the shear scalar $\sigma$, which leads to

$$
A=B^{k}
$$

where $k \neq 1$ is a positive constant. The physical reason for this assumption is warranted from the observations of the velocity redshift relation for extragalactic sources which suggest that the Hubble expansion of the universe may achieve isotropy when $\frac{\sigma}{\theta}$ is constant (Kantowski and Sachs [40]). Collins [41] have studied the physical significance of this condition for a perfect fluid.

Now from equations (12), (13) and (15), we get

$$
\frac{\ddot{B}}{B}+(k+1) \frac{\dot{B}^{2}}{B^{2}}+\frac{1}{(1-k) B^{2}}=0,
$$

By substituting $\dot{B}=f(B)$, the above equation becomes

$$
\frac{d f^{2}}{d B}+\frac{2(k+1)}{B} f^{2}=\frac{2}{(k-1) B}
$$

which admits a solution

$$
f^{2}=\left(\frac{d B}{d t}\right)^{2}=\frac{B^{2(k+1)}+c_{1}\left(k^{2}-1\right)}{B^{2(k+1)}\left(k^{2}-1\right)}
$$

where $c_{1}$ is an integrating constant. Now using the transformation $B=\mathcal{T}$ the metric (11) can be written as

$$
\begin{aligned}
d s^{2} & =\left(\frac{d t}{d B}\right)^{2} d B^{2}-B^{2 k} d r^{2}-B^{2}\left(d \psi^{2}+\sin ^{2} \psi d \varphi^{2}\right) \\
& =\frac{\mathcal{T}^{2(k+1)}\left(k^{2}-1\right)}{\mathcal{T}^{2(k+1)}+c_{1}\left(k^{2}-1\right)} d \mathcal{T}^{2}-\mathcal{T}^{2 k} d r^{2}-\mathcal{T}^{2}\left(d \psi^{2}+\sin ^{2} \psi d \varphi^{2}\right)
\end{aligned}
$$

From equations (12)-(14) and (19), we get scalar field potential as

$$
\mathcal{V}(\phi)=\frac{c_{1} k\left(k^{2}-1\right) \mathcal{T}^{-2(k+2)}}{4(4 \lambda+1)}-\frac{\left(3 k-2 k^{2}+3\right) \mathcal{T}^{-2}}{2(4 \lambda+1)\left(k^{2}-1\right)}
$$

and the scalar field $\phi$ as

$$
\phi=\int\left\{\frac{2 k \mathcal{T}^{-2}}{\epsilon(2 \lambda+1)\left(1-k^{2}\right)}-\frac{c_{1}(3 k+1) \mathcal{T}^{-2(k+2)}}{2 \epsilon(2 \lambda+1)(k+1)}\right\}^{\frac{1}{2}} d \mathcal{T}
$$

Substituting the equations (18), (20) and (21) in the function $f(R, T)=R+2 \lambda T$, we get

$$
\begin{aligned}
f(R, T)=\quad \mathcal{T}^{-2(k+2)}\left[2 c_{1}\left(k^{3}-3 k-1\right)+\frac{c_{1} \lambda(3 k+1)}{(2 \lambda+1)(k+1)}+\frac{2 c_{1} \lambda k\left(k^{2}-1\right)}{4 \lambda+1}\right] \\
+\frac{\mathcal{T}^{-2}}{k^{2}-1}\left[2 k\left(2 k^{2}+2 k-1\right)-\frac{2 k \lambda}{2 \lambda+1}-\frac{2 \lambda\left(3 k-2 k^{2}+3\right)}{4 \lambda+1}\right]
\end{aligned}
$$

Thus the metric (19) together with (20)-(22) constitutes Kanowski-Sachs scalar field cosmological model in $f(R, T)$ theory of gravity. 


\section{Physical discussion:}

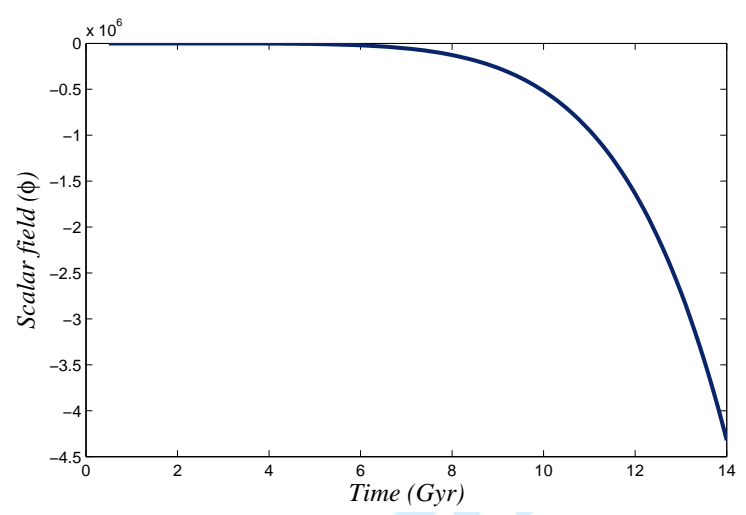

Figure 1: Plot of scalar field $(\phi)$ versus $\mathcal{T}$ for $k=1.1, c_{1}=2.8, \lambda=1$ and $\epsilon=1$.

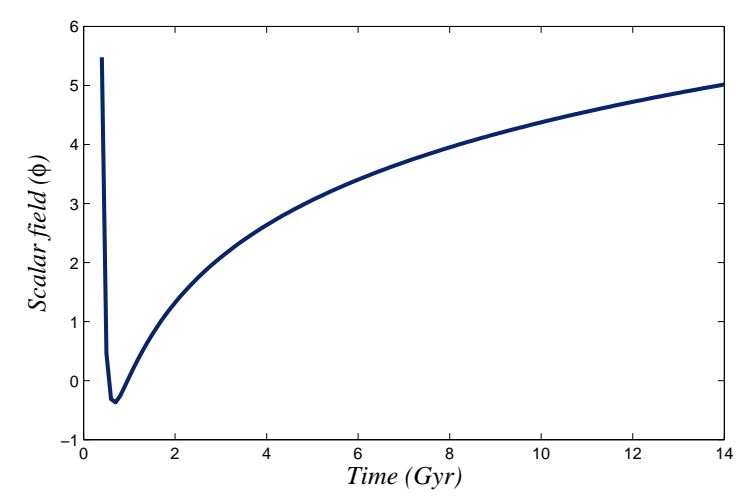

Figure 2: Plot of scalar field $(\phi)$ versus $\mathcal{T}$ for $k=1.1, c_{1}=2.8, \lambda=1$ and $\epsilon=-1$.

From Figs. 1 and 2, it can be seen that scalar field $\phi$ is varying in negative region throughout the evolution for quintessence model (i.e., for $\epsilon=1$ ). The scalar field corresponds to phantom $(\epsilon=-1)$ model which exhibits negative nature in the initial epoch, but positive at late times. It is quite interesting to mention here that the behavior of the scalar field is similar to the scalar field model obtained by Singh et al. [36]. Fig. 3 shows that scalar field potential is positive throughout the evolution of the universe.

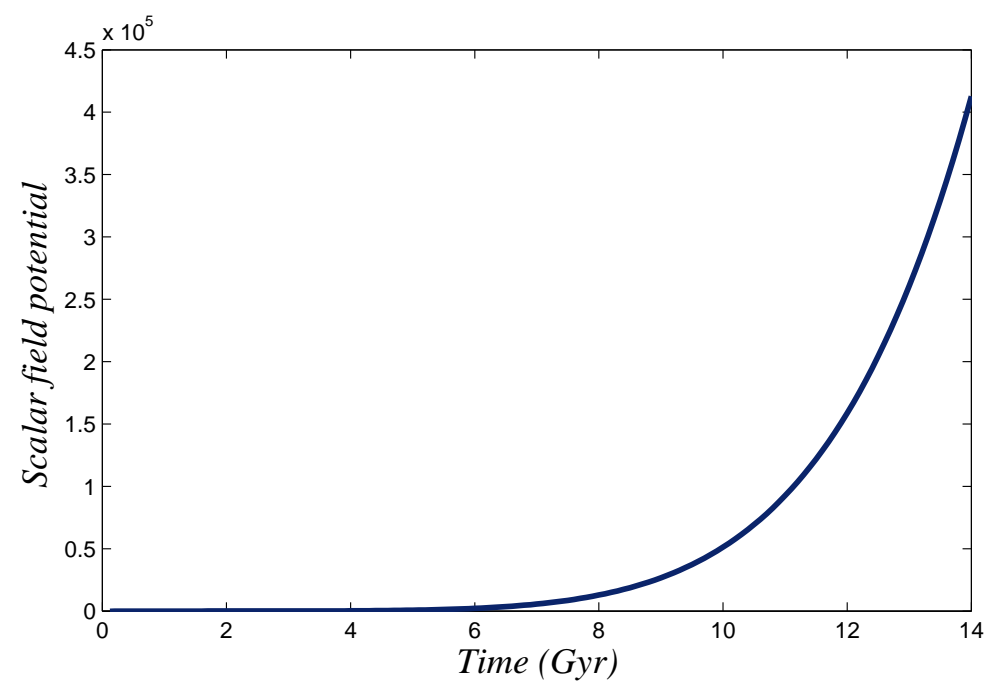

Figure 3: Plot of scalar field potential versus $\mathcal{T}$ for $k=1.1, c_{1}=2.8$ and $\lambda=1$.

We compute the following dynamical parameters which are significant in the physical discussion of the cosmological model (19): 
Volume and average scale factor are

$$
\begin{array}{r}
V=\sqrt{-g}=\mathcal{T}^{k+2} \sin \psi \\
a(t)=V^{\frac{1}{3}}=\left(\mathcal{T}^{k+2} \sin \psi\right)^{\frac{1}{3}} .
\end{array}
$$

The directional Hubble parameters and average Hubble parameter takes the form

$$
\begin{gathered}
H_{r}=k H_{\psi}=\frac{k \sqrt{\mathcal{T}^{2(k+1)}+c_{1}\left(k^{2}-1\right)}}{\mathcal{T}^{(k+2)} \sqrt{\left(k^{2}-1\right)}} \\
H=\frac{(k+2) \sqrt{\mathcal{T}^{2(k+1)}+c_{1}\left(k^{2}-1\right)}}{3 \mathcal{T}^{(k+2)} \sqrt{\left(k^{2}-1\right)}}
\end{gathered}
$$

Expansion scalar $(\theta)$ and shear scalar $\left(\sigma^{2}\right)$ can be obtained as

$$
\begin{aligned}
\theta & =3 H=\frac{(k+3) \sqrt{\mathcal{T}^{2(k+1)}+c_{1}\left(k^{2}-1\right)}}{\mathcal{T}^{(k+2)} \sqrt{\left(k^{2}-1\right)}} \\
\sigma^{2} & =\frac{1}{3}\left(H_{r}-H_{\psi}\right)^{2} \\
& =\frac{(k-1)\left(\mathcal{T}^{2(k+1)}+c_{1}\left(k^{2}-1\right)\right)}{(k+2)\left(\mathcal{T}^{2(k+1)}\right)}
\end{aligned}
$$

Average anisotropic parameter is

$$
\begin{aligned}
A_{h} & =\frac{1}{3}\left(\frac{\left(H_{r}-H\right)^{2}+2\left(H_{\psi}-H\right)^{2}}{H^{2}}\right) \\
& =6\left(\frac{k-1}{k+2}\right)^{2} .
\end{aligned}
$$

Deceleration parameter is given by

$$
\begin{aligned}
q & =\frac{-a \ddot{a}}{\dot{a}^{2}} \\
& =2-\frac{3(k+1) \mathcal{T}^{2(k+1)}}{(k+2)\left(\mathcal{T}^{2(k+1)}+c_{1}\left(k^{2}-1\right)\right)}
\end{aligned}
$$

Statefinder diagnostic: To differentiate various DE models Sahni et al. [42] introduced two 
new cosmological parameters $(r, s)$ named as statefinder parameters which are defined as

$$
\begin{aligned}
r= & \frac{\dddot{a}}{a H^{3}} \\
= & 10-\frac{27(k+1) \mathcal{T}^{2(k+1)}}{(k+2)\left(\mathcal{T}^{2(k+1)}+c_{1}\left(k^{2}-1\right)\right)}+\frac{18(k+1)^{2}\left(k^{4}-2 k^{2}+2\right) \mathcal{T}^{4(k+1)}}{(k+2)^{2}\left(\mathcal{T}^{2(k+1)}+c_{1}\left(k^{2}-1\right)\right)^{2}} \\
s= & \frac{r-1}{3\left(q-\frac{1}{2}\right)} \\
= & \left\{2(k+2)\left[c_{1}\left(k^{2}-1\right)-2 \mathcal{T}^{2(k+1)}\right]+\frac{4(k+1)^{2}\left(k^{4}-2 k^{2}+2\right) \mathcal{T}^{4(k+1)}}{(k+2)\left(\mathcal{T}^{2(k+1)}+c_{1}\left(k^{2}-1\right)\right)}\right\} \\
& X\left(c_{1}(k+1)\left(k^{2}-1\right)-k \mathcal{T}^{2(k+1)}\right)^{-1}
\end{aligned}
$$

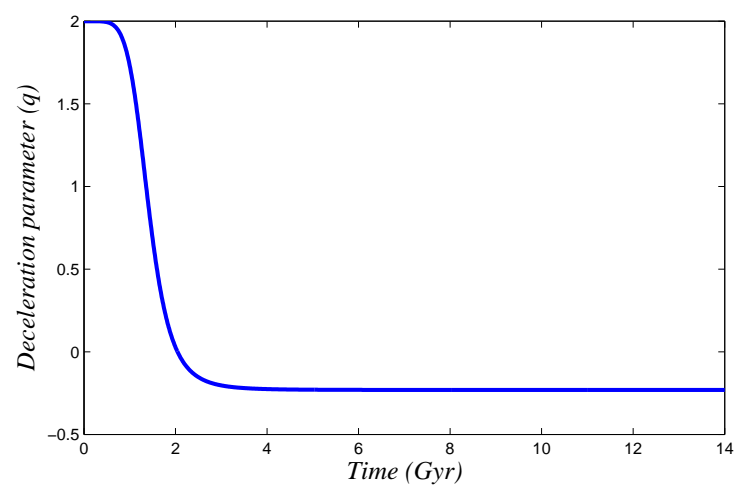

Figure 4: Plot of deceleration parameter versus $\mathcal{T}$ for $k=1.1$ and $c_{1}=2.8$.

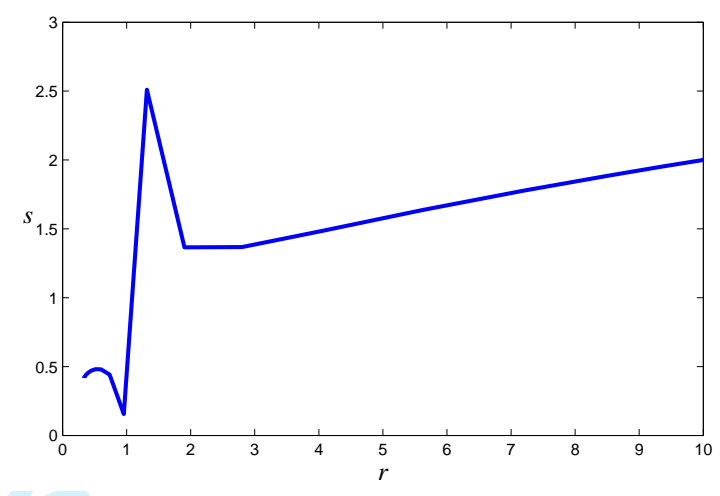

Figure 5: Plot of $r$ versus $s$ for $k=1.1$ and $c_{1}=2.8$.

The behavior of deceleration parameter $(q)$ against $\mathcal{T}$ has been depicted in Fig. 4 . It can be observed that the deceleration parameter varying from early decelerating phase (i.e., $q>0$ ) to present accelerating phase (i.e., $q<0)$. The $r-s$ statefinder plane for our model has been plotted in Fig. 5. The statefinder trajectories give us the quintessence and phantom regions, since $r<1$ and $s>0$.

Om-diagnostic: To discriminate different phases of the universe Sahni et al. [43] have introduced another tool named as Om-diagnostic. It is also used to distinguish the $\Lambda C D M$ for non-minimally coupled scalar field, quintessence model and phantom field through trajectories of the curves. The phantom DE era corresponds to the positive trajectory, whereas the negative trajectory means that DE constitutes quintessence. The Om-diagnostic in terms of $x=\ln (1+z)^{-1}$ function is 
defined as

$$
\begin{aligned}
O m(x) & =\frac{H^{2}(x)-H_{0}}{H_{0}\left(x^{3}-1\right)} \\
& =\left(x^{3}-1\right)^{-1}\left[\frac{(k+2)^{2}\left(\mathcal{T}_{0}^{2(k+1)} \exp \left(\frac{6 x(k+1)}{k+2}\right)+c_{1}\left(k^{2}-1\right)\right)}{H_{0} \mathcal{T}_{0}^{2(k+2)}\left(k^{2}-1\right) \exp (6 x)}-1\right]
\end{aligned}
$$

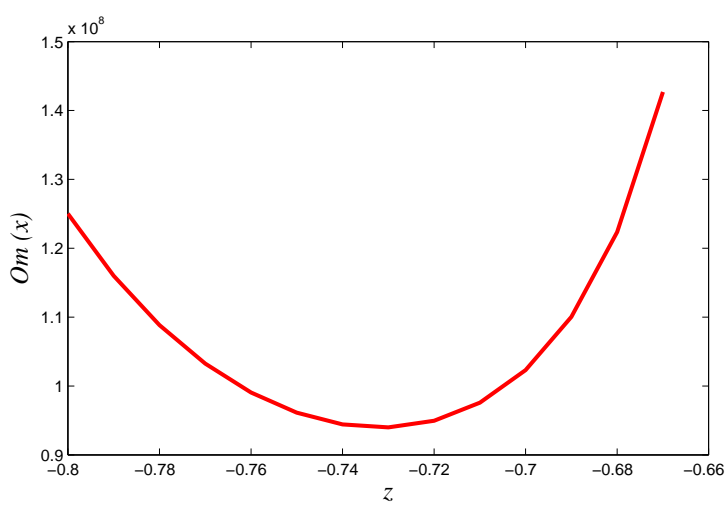

Figure 6: Plots of Om-diagnostics versus redshift $(z)$ for $k=1.1, H_{0}=72$ and $c_{1}=2.8$.

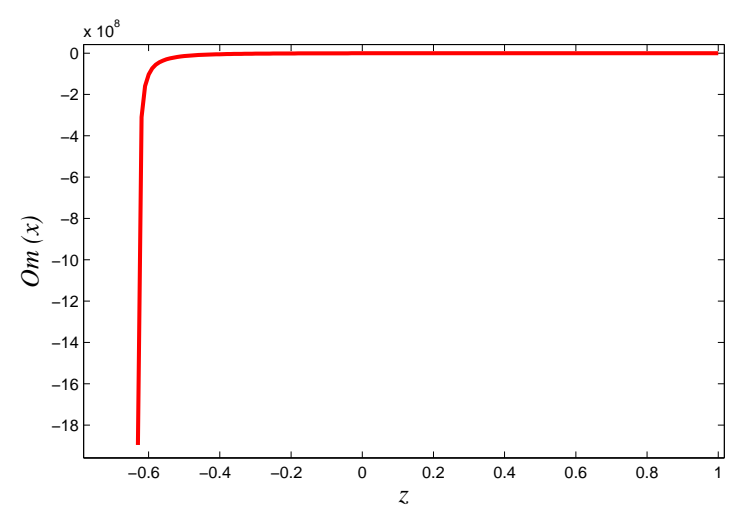

Figure 7: Plots of Om-diagnostics versus redshift $(z)$ for $k=1.1, H_{0}=72$ and $c_{1}=2.8$.

In Figs. 6 and 7 we plotted the Om-diagnostic in terms of $z$ by taking $x=\ln (1+z)^{-1}$. From Fig. 6 it can be observed that the trajectory of the Om-diagnostic plane is positive, which implies phantom behavior. However, the trajectory present negative slope in Fig. 7, which represents the quintessence behavior of the universe. This type of behavior is consistent with recent observational data.

Look-back time: The look-back time $\Delta \mathcal{T}=\mathcal{T}_{0}-\mathcal{T}$ to an object is the difference between the age $\mathcal{T}_{0}$ of the universe now (at observation) and the age $\mathcal{T}$ of the universe at the time the photons were emitted (according to the object). It is used to predict properties of high-redshift objects with evolutionary models, such as passive stellar evolution for galaxies. The look-back time is defined by Arbab [44], Hogg [45] and Rudra [46] as

$$
\Delta \mathcal{T}=1+z=\int_{a_{0}}^{a} \frac{d a}{\dot{a}}
$$

where $a_{0}$ is the present value of the scale factor of the universe and can be obtained from equation (24) at $\mathcal{T}=\mathcal{T}_{0}$. The redshift $z$ can be defined by

$$
\frac{a_{0}}{a}=1+z=\left(\frac{\mathcal{T}_{0}}{\mathcal{T}}\right)^{\frac{k+2}{3}}
$$


which gives the look-back time in the following form

$$
\mathcal{T}-\mathcal{T}_{0}=\mathcal{T}_{0}\left((1+z)^{\frac{-3}{k+2}}-1\right)
$$

Clearly from equation (36), early universe is represented by $z \rightarrow \infty$ implies $\mathcal{T} \rightarrow 0$ (since $k>0$ ) and late universe $z \rightarrow-1$, which implies $\mathcal{T} \rightarrow \infty$. Also $z \rightarrow 0$ gives the present age $\mathcal{T}=\mathcal{T}_{0}$ of the universe.

Luminosity distance: The luminosity distance $\left(d_{L}\right)$ of a light source is derived as the ratio of detected energy flux, $L$ and the apparent luminosity $l_{*}$ i.e., $d_{L}^{2}=\frac{L}{4 \pi l_{*}}$. This is a way of expanding the light coming from a distant object. It is not the actual distance because inverse square law does not hold in real universe. To calculate the luminosity distance the inverse square law of brightness is generalized from static Euclidean space to an expanding curved space by the following expression (Waga [47])

$$
d_{L}=a_{0}(1+z) r_{1}(z)
$$

where $r_{1}(z)$ is the radial coordinate distance of the object at light emission and is given by

$$
r_{1}(z)=\int_{\mathcal{T}}^{\mathcal{T}_{0}} \frac{d \mathcal{T}}{a}
$$

From equations (24), (37) and (38), we get

$$
d_{L}=\frac{3 a_{0} \mathcal{T}^{\frac{k+5}{3}}}{k+2}\left[(1+z)-(1+z)^{\frac{-3}{k+2}}\right]
$$

Angular diameter distance: It is used to convert angular separations in telescope images into proper separations at the source. It is famous for not increasing indefinitely as $z \rightarrow \infty$; it turns over at $z \approx 1$ and thereafter more distant objects actually appear larger in angular size. The angular diameter of a light source with proper distance $\left(r_{1}(z)\right) D$ at $\mathcal{T}_{0}$ is define by $\operatorname{Hogg}[45]$ and Rudra [46]

$$
\delta=\frac{D(1+z)^{2}}{d_{L}} .
$$

Now the angular diameter distance $d_{A}$ is defined as the ratio of the source diameter to its angular diameter (in radians)

$$
d_{A}=\frac{D}{\delta}=d_{L}(1+z)^{-2}
$$

For our model angular diameter distance $\left(d_{A}\right)$ is given by

$$
d_{A}=\frac{3 a_{0} \mathcal{T}^{\frac{k+5}{3}}}{k+2}\left[(1+z)^{-1}-(1+z)^{\frac{-(2 k+7)}{k+2}}\right]
$$


Distance modulus: The distance modulus $\left(D_{M}\right)$ is given by

$$
\begin{aligned}
D_{M} & =5 \log \left(d_{L}\right)+25 \\
& =5 \log \left(\frac{3 a_{0} \mathcal{T}^{\frac{k+5}{3}}}{k+2}\left[(1+z)-(1+z)^{\frac{-3}{k+2}}\right]\right)+25
\end{aligned}
$$

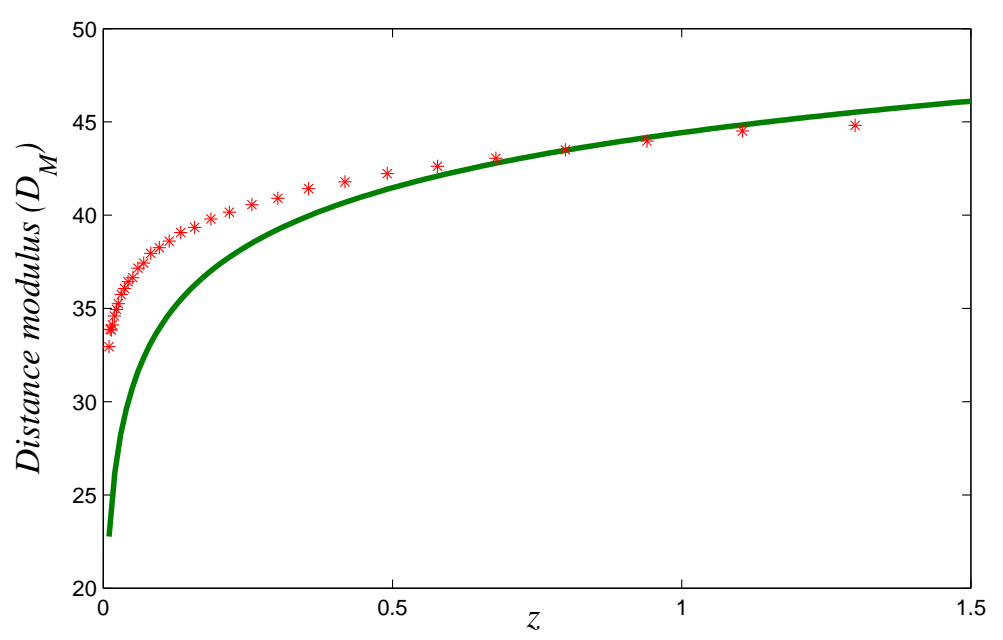

Figure 8: Plot of distance modulus versus redshift to the obtained model compared with SDSS-II and SNLS supernova data from Betoule et al. [48].

In Fig. 8, we compare the obtained model and joint analysis of the SDSS-II and SNLS supernova samples data. The dots represent the observed distance modulus by SDSS-II and SNLS supernova data where as solid line represents the distance modulus $D_{M}$ of the obtained model. It can be observed that the obtained model coincides with observational data at high redshift values.

In case of $c_{1}=0$, from equation (18) the metric (19) can be written as

$$
d s^{2}=d t^{2}-\frac{t^{2 k}}{\left(k^{2}-1\right)^{k}} d r^{2}-\frac{t^{2}}{\left(k^{2}-1\right)}\left(d \psi^{2}+\sin ^{2} \psi d \varphi^{2}\right)
$$

the scalar field and scalar potential are given by

$$
\begin{aligned}
\phi & =\sqrt{\frac{-2 k}{\epsilon(2 \lambda+1)}} \log (t) \\
\mathcal{V}(\phi) & =\frac{k(k+1)}{(4 \lambda+1) t^{2}} .
\end{aligned}
$$


Other physical properties are obtained as

$$
\begin{aligned}
H & =\frac{k+2}{3 t} \\
\theta & =\frac{k+2}{t} \\
\sigma^{2} & =\frac{(k-1)^{2}}{3 t^{2}} \\
q & =\frac{1-k}{k+2}
\end{aligned}
$$

It is clear from equation (45) that the phantom scalar field (i.e., $\epsilon=-1$ ) only exists. The scalar field $(\phi)$ and scalar potential $(\mathcal{V}(\phi))$ becomes infinity initially and gradually decrease then finally tends to zero at late times. The model (44) has a constant deceleration parameter and it represents accelerated expansion for $k>1$. From equations (48) and (49), we can observe that $\frac{\sigma}{\theta}$ is constant and hence the model (45) is anisotropic.

\section{Summary and conclusions:}

$f(R, T)$ theory of gravity can be treated as a possible candidate in explaining the role of DE in the accelerating universe. A suitable form of Lagrangian which can explain the cosmic evolution in a definite way is still under consideration. In this study we have obtained solution of the $f(R, T)$ field equations with scalar field for Kantowski-Sachs space time.

The volume of the model increases as $\mathcal{T}$ increases subject to the condition $k>0$. It can be seen that Hubble parameter, expansion scalar and shear scalar are dynamical and approach to zero as $\mathcal{T} \rightarrow \infty$. Moreover, for earlier times (i.e., as $\mathcal{T} \rightarrow 0$ ) these parameters take infinitely large values. So the model starts its evolution with zero volume at $\mathcal{T}=0$ with infinite rate of expansion. But this expansion rate is slowing down in its evolution. Since the average anisotropic parameter $A_{h} \neq 0$ for $k \neq 1$ the model does not approach isotropy. The scalar field $\phi$ is negative for quintessence model, whereas it varies in positive region for phantom model. The scalar field potential is positive throughout the evolution of the universe. From the plot of deceleration parameter we can observe that there is a smooth transition of the universe from early decelerating phase to present accelerating epoch, which is in accordance with the modern observational data. The statefinder parameters and Om-diagnostic both describe quintessence and phantom eras of the evolving universe. The distance modulus curve of the model is in nice agreement with the SDSS-II and SNLS supernova data for high redshift value. This imply that the obtained scalar field model is physically realistic. Finally different types of cosmological distance measurements have been studied for the obtained model. For $c_{1}=0$ the model (19) reduces to the model with constant deceleration parameter. It is worth mentioning here that in this case phantom scalar field model only exists. 


\section{References}

[1] S.Perlmutter, et al.: Astrophys. J. 517, 565 (1999)

[2] D.N.Spergel, et al.: Astrophys. J. Suppl. Ser. 148, 175 (2003)

[3] M.Tegmark, et al.: Phys. Rev. D 69, 103501 (2004)

[4] A.G.Riess, et al.: Astrophys. J. 659, 98 (2007)

[5] C.Fedeli, L.Moscardini, M.Bartelmann: Astron. Astrophys. 500, 667 (2009)

[6] R.R.Caldwell: Phys. Lett. B 23, 545 (2002)

[7] S.Nojiri, S.D.Odintsov: Phys. Lett. B 147, 562 (2003)

[8] V.Sahni, A.A.Starobinsky: Int. J. Mod. Phys. D 9, 373 (2000)

[9] V.Sahni: Lect. Notes Phys. 653, 141 (2004)

[10] T.Padmanabhan: Gen. Relativ. Gravit. 40, 529 (2008)

[11] O.Akarsu, B.C.Kilinc: Gen. Relativ. Gravit. 42, 119 (2010)

[12] M.Sharif, M.Zubair: Astrophys. Space Sci. 330, 399 (2010)

[13] S.Capozziello, M. Francaviglia: Gen. Relativ. Grav. 40, 357 (2008)

[14] S.Nojiri, S.D.Odintsov: Phys. Rep. 505, 59 (2011)

[15] S.Nojiri, S.D.Odintsov, M.Sami: Physical Rev. D 74, 046004 (2006)

[16] E.V.Linder: Phys. Rev. D 81, 127301 (2010)

[17] T.Harko, et al.: Physics review D 84, 024020 (2011)

[18] K.S.Adhav: Astrophys. Space Sci. 339, 365 (2012)

[19] D.R.K.Reddy, R.Santi Kumar: Astrophys. Space Sci. 344, 253 (2013)

[20] B.Mishra, P.K.Sahoo: Astrophys. Space Sci. 352, 331 (2014)

[21] V.U.M.Rao, D.Neelima: Astrophys. Space Sci. 345, 427 (2013)

[22] V.U.M.Rao, et al.: Eur. Phys. J. Plus 129, 17 (2014)

[23] M.J.S.Houndjo: Int. J. Mod. Phys. D 21, 1250003 (2012)

[24] Shri Ram, S.Chandel: Astrophys Space Sci. 355, 195 (2015) 
[25] V.U.M.Rao, et al.: Prespacetime Journal, 6, 947 (2015)

[26] Y.Aditya, et al.: Astrophys. Space Sci. 361, 56 (2016)

[27] K.A.Olive: Phys. Rev. 190, 307 (1990)

[28] O.Bertolami: Nuovo Cimento Soc. Ital. Fis. 93B, 36 (1986)

[29] B.Ratra, P.J.E.Peebles: Phys. Rev. D 37, 3406 (1988)

[30] R.R.Caldwell, R.Dave, P.J.Steinhardt: Phys. Rev. Lett. 80, 1582 (1998)

[31] O.Bertolami, J.Paramos: Classical Quantum Gravity 21, 3309 (2004)

[32] M.Sharif, M.Zubair: J. Phys. Soc. Jpn. 81, 114005 (2012)

[33] C.P.Singh, V.Singh: Mod. Phy. Lett. A 26, 1495 (2011)

[34] M.Sharif, Abdul Jawad: Commun. Theor. Phys. 60, 183 (2013)

[35] V.Singh, C.P.Singh: Astrophys. Space Sci. 355, 2183 (2014)

[36] G.P.Singh, et al.: Chinese Journal of Physics 54, 244 (2016)

[37] K.S.Thorne: ApJ. 148, 51 (1967)

[38] G.C.Samanta: Int. J. Theor. Phys. 52, 2647 (2013)

[39] D.R.K.Reddy, et al.: Eur. Phys. J. Plus 129, 96 (2014)

[40] R.Kantowski, R.K.Sachs: J. Math. Phys. 7, 433 (1966)

[41] C.B.Collins, et al.: Gen. Relativ. Gravit. 12, 805 (1980)

[42] V.Sahni, et al.: JETP Lett. 77, 201 (2003)

[43] V.Sahni, A.Shafieloo, A.A.Starobinsky: Phys. Rev. D 78, 103502 (2008)

[44] A.I.Arbab: arXiv:astro-ph/9810239 (1998)

[45] D.W.Hogg: (2000). arXiv:astro-ph/9905116v4

[46] P.Rudra: Astrophys. Space Sci., 342, 579 (2012)

[47] I.Waga: Astrophys. J. 414, 436 (1993)

[48] M.Betoule, et al.: Astronomy and Astrophysics 568, A22 (2014) 